\title{
Effects of Acute Whole-Body Vibration Practice on Maximal Fat Oxidation in Adult Obese Males: A Pilot Study
}

\author{
Gian Pietro Emerenziani $^{a} \quad$ Dafne Ferrari $^{b}$ Simona Fittipaldi ${ }^{c}$ \\ Viviana Maria Bimonte $^{d}$ Chiara Marocco $^{b}$ Emanuela A. Greco ${ }^{d}$ \\ Fabrizio Perroni $^{\mathrm{e}} \quad$ Silvia Migliaccio $^{\mathrm{b}}$ Andrea Lenzi $^{\mathrm{d}}$ Carlo Baldari $^{f}$ \\ Laura Guidetti ${ }^{b}$

\begin{abstract}
aDepartment of Experimental and Clinical Medicine, "Magna Græcia" University, Catanzaro, Italy; ${ }^{b}$ Department of Movement, Human and Health Sciences, Section of Health Sciences,

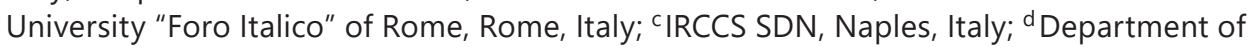
Experimental Medicine, Section of Medical Pathophysiology, Endocrinology and Nutrition,

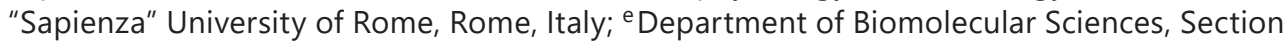
of Exercise and Health Sciences, "Carlo Bo" University of Urbino, Urbino, Italy; ${ }^{\dagger}$ Campus University, Novedrate, Italy
\end{abstract}

\section{Keywords}

Obesity · Exercise $\cdot$ Endocrine regulation of energy balance $\cdot$ Fat oxidation · Vibration stimulus

\section{Abstract}

Objective: Whole-body vibration (WBV) training has been established as a useful method to improve physical fitness in obese individuals. However, the effects of WBV exercise on maximal fat oxidation (MFO) have not been examined in obese subjects yet. Method: MFO was evaluated during a cardiopulmonary exercise test (CPET) on a treadmill in 12 adult obese males $\left(B M I=34.9 \pm 3.3 \mathrm{~kg} / \mathrm{m}^{2}\right)$ after three different warm-up conditions: static half squat plus WBV (HSV), static half squat without WBV (HSWV), and rest (REST). Cortisol levels were evaluated before and after the warm-up, and $1 \mathrm{~min}$ (T1), $10 \mathrm{~min}$ (T10), and $30 \mathrm{~min}$ (T30) of the recovery phase. Results: MFO was significantly higher in HSV $(p=0.013 ; 569.4 \pm 117.9 \mathrm{mg} / \mathrm{min})$ and $\operatorname{HSWV}(p=0.033 ; 563.8 \pm 142.9 \mathrm{mg} / \mathrm{min})$ than REST $(445.5 \pm 117.9 \mathrm{mg} / \mathrm{min})$. Cortisol concentrations at T1 were significantly higher in $\operatorname{HSV}(p=0.023)$ and HSWV $(p=0.015)$ than REST. Moreover, cortisol concentrations were significantly lower at T30 than T1 in HSWV $(p=0.04)$. No differences were found between T30 and T1 in HSV. Conclusions: Active warm-up increases MFO; however, vibration stimulus during half squatting does not increase MFO during a CPET in obese subjects. The lack of significant differences of cortisol concentrations in HSV during the recovery phase might suggest a long-term effect of WBV on the endocrine system. 
Emerenziani et al.: Whole-Body Vibration and Maximal Fat Oxidation in Obesity

\section{Introduction}

Obesity is a condition characterized by an abnormal or excessive adipose tissue accumulation that may impair health [1]. Worldwide obesity has nearly tripled since 1975, and in 2016 over 650 million of people aged 18 years and older were obese [1]. Obesity impairs health leading to an increased risk of morbidity and mortality [2]. According to the World Health Organization [1], the obesity condition can be preventable. Indeed, it is well known that regular physical activity (PA) and a balanced diet are an important strategy for body weight balance and weight control. In particular, regular individualized PA and diet have been shown to increase cardiocirculatory fitness, body composition, and quality of life $[3,4]$. Despite these positive effects, obese subjects are inclined to be sedentary, and the high dropout rate after a period of aerobic training confirms that these subjects are not willing to be included in regular exercise programs [4].

In obese subjects, an individualized exercise program should also aim to lose weight; therefore, it is very important to consider all the variables that might improve maximal fat oxidation (MFO) rates. Several international groups have focused their research on the evaluation and characterization of the exercise intensity that may induce and improve the MFO [5-7]. Exercise intensity at which MFO occurs is termed Fat max $_{\text {, }}$ and it is influenced by several factors, such as type of warm-up, mode of exercise (cycling, walking, or running), subjects' age, sex, and fitness level [8,9]. Interestingly, no previous studies focused on the effects of active warm-up on MFO in obese subjects.

In the last years, whole-body vibration (WBV), which is applied using a vibration platform on which a subject stands performing exercise while receiving a vibratory stimulus, has become a common exercise used as alternative exercise modality to improve physical fitness and physical performance in obese [10] and non-obese [11] subjects. Several studies showed that long-term WBV practice has positive effects on body composition, bone mineral density, and heart rate (HR) variability $[12,13]$. Moreover, it is well established that a single session of WBV positively influences the anabolic hormones in aged individuals [14], and in obese subjects [15]. As previously reported [16], WBV alone stimulates growth hormone (GH) release in severely obese female subjects [16] confirming that WBV practice might have an effect on the hypothalamic-pituitary-adrenal (HPA) axis [15].

One of the physiological effects of GH is the stimulation of lipolysis in adipocytes [17], and a study has shown an increase in fuel utilization after acute GH administration [18]. Therefore, it could be hypothesized that acute WBV during warm-up, which leads to an increase of GH levels, might increase fat oxidation during exercise in obese men. While the literature highlights the positive effects of WBV on physical fitness and body composition, there are no data on the effects of acute WBV on MFO in obese adults. Therefore, the aims of this study were: to evaluate whether WBV during active warm-up might increase MFO during exercise in obese adults and to assess the physiological and endocrinological responses to different warm-up protocols in obese adults.

\section{Materials and Methods}

\section{Subjects}

Twelve obese men (age $=43.6 \pm 10.9$ years) among individuals admitted to the Day Hospital of the Department of Experimental Medicine, Section of Medical Pathophysiology, Endocrinology and Nutrition, Policlinico Umberto I, "Sapienza University" of Rome, were recruited for this study. All participants underwent clinical examination to exclude any contraindications to PA. Blood samples were collected in the morning after an overnight fast 
Emerenziani et al.: Whole-Body Vibration and Maximal Fat Oxidation in Obesity

Table 1. Anthropometric characteristics, metabolic and hormonal parameters of the subjects involved in the study

\begin{tabular}{lcc}
\hline Variable & Value & Normal range \\
\hline Weight, kg & $97.7 \pm 14.5$ & \\
Height, m & $1.67 \pm 0.12$ & \\
BMI, kg/m² & $34.9 \pm 3.3$ & \\
Fat mass, \% & $35.4 \pm 5.7$ & \\
Fat-free mass, kg & $63.4 \pm 11.8$ & \\
Glycemia, mg/dL & $100.7 \pm 23.1$ & $70.3-100.9$ \\
Insulinemia, $\mu \mathrm{U} / \mathrm{mL}$ & $10.0 \pm 4.7$ & $2.6-24.9$ \\
Homa index, score & $2.7 \pm 1.5$ & $<2.5$ \\
\hline Hb1Ac, \% & $5.8 \pm 0.8$ & $4-5.9$ \\
Total cholesterol, mg/dL & $188.5 \pm 26.1$ & $81-235$ \\
HDL, mg/dL & $51.8 \pm 13.9$ & $34-56$ \\
LDL, mg/dL & $117.9 \pm 20.7$ & $54.8-120$ \\
Triglycerides, mg/dL & $88.7 \pm 26.1$ & $40-235$ \\
GH, ng/mL & $0.5 \pm 0.6$ & $0.05-16$ \\
TSH, $\mu U$ /mL & $2.2 \pm 0.8$ & $0.27-4.2$ \\
IGF-1, ng/mL & $116.2 \pm 32.7$ & $94-269$ \\
Testosterone level, ng/mL & $3.1 \pm 0.9$ & $2.8-8$ \\
\hline
\end{tabular}

Data are presented as mean \pm SD. Hb1Ac, glycated hemoglobin; HDL, high-density lipoprotein cholesterol; LDL, low-density lipoprotein cholesterol; GH, growth hormone; TSH, thyroid-stimulating hormone; IGF-1, insulin growth factor-1.

to evaluate glycemia, insulinemia, glycated hemoglobin (Hb1Ac), total cholesterol, highdensity lipoprotein cholesterol, low-density lipoprotein cholesterol, triglycerides, GH, thyroid-stimulating hormone, insulin growth factor-1 (IGF-1), and testosterone levels. Subjects' anthropometric characteristics and metabolic and hormonal parameters are depicted in Table 1.

The inclusion criteria were adult age (45-65 years), BMI $\geq 30 \mathrm{~kg} / \mathrm{m}^{2}$, and fat mass $>25 \%$ in males. The exclusion criteria were neuropathy, autonomic dysfunction, cardiovascular diseases (myocardial infarction during the previous 6 months, myocardial ischemia or ventricular tachycardia, obstructive valvular heart disease), uncontrolled hypertension (blood pressure values exceeding $140 \mathrm{~mm} \mathrm{Hg}$ systolic or $90 \mathrm{~mm} \mathrm{Hg}$ diastolic), thyroid diseases including autoimmunity, or any treatment with thyroid hormone preparations or amiodarone, methimazole, or propylthiouracil in the prior 3 months. During clinical examination, all subjects reported to be sedentary and not being engaged in any organized PA.

All subjects provided written informed consent before the beginning of the study. This study was conducted according to the Declaration of Helsinki and approved by the Sapienza University of Rome Ethical Committee (approval No. 70/11, 2011).

\section{Experimental Procedures}

All subjects performed one pre-testing session and 3 testing sessions. During the pretesting session, anthropometrics and body composition variables were assessed, after which subjects underwent a preliminary exercise test to get familiarized with the procedures. Body weight and height were measured using a scale and a stadiometer to the nearest $0.1 \mathrm{~kg}$ and $0.1 \mathrm{~cm}$, respectively. Body composition was measured by a bioelectrical impedance method (BIA AKERN 101 Anniversary, Pontassieve, Italy) while the subjects wore minimal clothing (i.e., underwear), in the thermoneutral state and 90 min after breakfast. Breakfast energy intake was standardized for all subjects and set at approximately $20-25 \%$ of total daily energy 
Emerenziani et al:: Whole-Body Vibration and Maximal Fat Oxidation in Obesity

expenditure. Total daily energy expenditure was determined by adding the resting metabolic rate and PA level. The resting metabolic rate was estimated by the Bernstein equation [19], while PA level was estimated by the International Physical Activity Questionnaire (IPAQ) [20]. After the familiarization session, the study adopted a randomized crossover design consisting of three measurements of MFO after three different warm-up conditions on separate days in random order and separated by at least 1 week. In the half squat vibration (HSV) condition, subjects remained on a Pro Evolve vibrating platform (DKN, USA) in a static half squat position for $72 \mathrm{~s}$. The vibration stimulus was repeated at the same condition for 10 consecutive series with a 50-s rest in between [16]. In half squat without the vibration stimulus (HSWV) condition, subjects performed the same warm-up protocol of the HSV condition but without the vibration stimulus. In the rest condition (REST), subjects did not perform any active or passive warm-up, and they sat on a chair. After 3 min from warm-up conditions, all subjects performed a cardiopulmonary exercise test (CPET) on a treadmill to evaluate peak oxygen uptake $\left(\mathrm{VO}_{2 \text { peak }}\right)$ and MFO. During all the three testing sessions, subjects' HR (beats $\times$ min) was continuously recorded using an HR monitor (RS 400, Polar Electro ${ }^{\mathrm{TM}}$, Kempele, Finland). Lactate values were assessed before the warm-up (PRE), and 1 min (T1), 3 min (T3), 6 min (T6), and 10 min (T10) after CPET (recovery phase). Salivary concentrations were assessed before the warm-up (PRE), after the warm-up (POST), and 1 min (T1), $10 \mathrm{~min}$ (T10), and $30 \mathrm{~min}$ (T30) after CPET (recovery phase). All subjects were tested in the morning (between 9:00 and 12:00 a.m.) and under similar environmental conditions (temperature $21-22^{\circ} \mathrm{C}$; humidity $50-60 \%$ ). Subjects had their usual breakfast at least $1.5 \mathrm{~h}$ before the test session.

\section{Cardiorespiratory Evaluation}

A CPET on a treadmill (Woodway PRO, Woodway, Waukesha, WI, USA) was used to evaluate the peak oxygen uptake $\left(\mathrm{VO}_{2 \text { peak }}\right)$. Breath by breath measures of $\mathrm{VO}_{2}$, carbon dioxide production $\left(\mathrm{VCO}_{2}\right)$, and minute ventilation (VE) were measured (Quark RMR-CPET Cosmed ${ }^{\mathrm{TM}}$, Rome, Italy) [21]. The system was calibrated, before each test, according to the manufacturer's instructions. In detail, the two-point gas calibration was completed sampling the ambient air and the gas from a certified tank containing $16 \% \mathrm{O}_{2}, 5 \% \mathrm{CO}_{2}$ and standard atmospheric nitrogen. The CPET on treadmill protocol started at $3 \mathrm{~km} / \mathrm{h}$, then the speed was increased by $1 \mathrm{~km} / \mathrm{h}$ every $3 \mathrm{~min}$ until a speed of $5 \mathrm{~km} / \mathrm{h}$ was reached. Then, the slope was increased by $2 \%$ every 3 min until subjects declared to perceive a fatigue corresponding to a value of 10 on RPE-OMNI-Walk/Run Scale [22]. The 3-min step exercise protocol on a treadmill has been used previously to determine substrate oxidation in obese population [23]. After CPET, all subjects performed an active recovery lasting $3 \mathrm{~min}$ at the speed set at 3 $\mathrm{km} / \mathrm{h}$ plus a passive recovery (sit position) lasting $27 \mathrm{~min}$. Therefore, the recovery phase lasted $30 \mathrm{~min}$. During the test, the highest $\mathrm{VO}_{2}$ attained was chosen as the $\mathrm{VO}_{2 \text { peak. }}$. During the graded exercise test, all subjects reach a value of respiratory exchange ratio (RER) equal to 1.0. Subjects' $\mathrm{HR}_{\max }$ was calculated using the following equation: 208-0.7 $\times$ age [24]. A standard definition of the OMNI-Walk/Run Scale was explained to the subjects immediately before the exercise test. During exercise testing, subjects were asked to report the degree of exertion on the OMNI-Walk/Run scale every $3 \mathrm{~min}$.

\section{Indirect Calorimetry and Calculations}

The average values for $\mathrm{VO}_{2}(\mathrm{~mL} / \mathrm{min})$ and $\mathrm{VCO}_{2}(\mathrm{~mL} / \mathrm{min})$, obtained during the graded exercise test, were calculated over the last $2 \mathrm{~min}$ of each stage [25]. Fat oxidation rate was calculated by using the stoichiometric equation $\left(\mathrm{Fat}_{\mathrm{oxi}}[\mathrm{mg} / \mathrm{min}]=1.695 \times \mathrm{VO}_{2}-1.701 \times \mathrm{VCO}_{2}\right.$ $-1.77 \times \mathrm{n}$ ) for all exercise intensities, with the assumption that urinary nitrogen (n) excretion was negligible $(n=0)$ [5]. For each subject, a best-fit polynomial curve was constructed using 
fat oxidation rate (expressed as $\mathrm{mg} / \mathrm{min}$ ) versus exercise intensity (expressed as $\mathrm{HR}$ and $\mathrm{VO}_{2}$ ). The peak rate of fat oxidation measured over the entire range of exercise intensities (MFO), and the exercise intensity at which the MFO was observed (Fat ${ }_{\max }$ ) were calculated for all subjects.

\section{Saliva Sample Collections}

Saliva samples were collected using a saliva-collecting tube (Salivette; Sarstedt, Germany) before the warm-up (PRE), immediately after the warm-up conditions (HSV and HSWV) (POST) at $1 \mathrm{~min}$ (T1), $10 \mathrm{~min}$ (T10), and $30 \mathrm{~min}$ (T30) after the CPETs.

To avoid the risk of saliva contamination, all participants were instructed not to eat or drink 90 min prior to saliva collection. Saliva samples were collected between 9:00 and 12: 00 a.m.

After collection, saliva-collecting tubes were centrifuged at $2,000 \mathrm{~g}$ for $15 \mathrm{~min}$ (at $4{ }^{\circ} \mathrm{C}$ ), and samples were stored at $-20^{\circ} \mathrm{C}$ prior to hormone analysis.

Salivary cortisol concentration was measured using a commercially available ELISA kit (IBL International GmbH, Hamburg, Germany). All samples were analyzed in duplicate in the same assay. The test was performed, according to the manufacturer's protocol. The sensitivity of the cortisol assay was $0.05 \mathrm{ng} / \mathrm{mL}$ (range of detection: $0.15-30 \mathrm{ng} / \mathrm{mL}$ ) with an interassay coefficient of variation from 3.2 to $6.1 \%$.

\section{Statistical Analysis}

The SPSS statistical package (Version 23.0 for Windows; SPSS Inc., Chicago, IL, USA) was used for data analysis and presented as mean \pm SD. Power analysis calculations $\left(G^{*}\right.$ Power 3.1.9.2 software) using a power of 0.8 and a medium effects size of 0.4 revealed that a sample of 12 subjects will provide sufficient power for our analysis. Statistical significance was set at $p \leq 0.05$. Before further analysis, normal distribution of the dependent variables was tested by applying the Kolmogorov-Smirnov test. This test showed that the cortisol variable had skewed distributions and different physiological variables were normally distributed. Therefore, for the cortisol variable the statistical analysis was based on the nonparametric Friedman's test followed, whenever significant, by Wilcoxon test for matched pairs as post hoc analysis. ANOVA for repeated measures was used to detect significant effects of warm-up conditions (HSV, HSWV, REST) on MFO and cardiorespiratory variables. The lactate variable was analyzed using a 3 (warm-up conditions) $\times 5$ (times of measurement) repeated measures ANOVA. After identifying any main effects and interaction, post hoc comparisons were carried out using a paired $t$ test with Bonferroni adjustment.

\section{Results}

Warm-Up and Cardiopulmonary Exercise Test

The first set of results showed that there were no significant differences in mean HR $\left(\mathrm{HR}_{\text {mean }}\right)$ and RPE between the HSV and HSWV warm-up conditions. Instead, maximal HR $\left(\mathrm{HR}_{\max }\right)$ during HSV was significantly $(p=0.03$ ) higher than HSWV (Table 2).

Regarding the CPET results, no significant differences were found for $\mathrm{VO}_{2}$ and $\mathrm{HR}$ at peak. Instead, at $\mathrm{Fat}_{\max } \mathrm{VO}_{2}$ and $\% \mathrm{HR}_{\max }$ were significantly higher in $\mathrm{HSV}(p=0.021$ and $p=0.001$ for $\mathrm{VO}_{2}$ and $\mathrm{HR}$, respectively) and $\mathrm{HSWV}\left(p=0.035\right.$ and $p=0.019$ for $\mathrm{VO}_{2}$ and HR, respectively) than REST (Table 2). No differences were found between $\mathrm{HSV}$ and $\mathrm{HSWV}$ for $\mathrm{VO}_{2}$ and $\mathrm{HR}$ variables at peak and at $\mathrm{Fat}_{\max }$. 
Fig. 1. Maximal fat oxidation during the cardiopulmonary exercise test after three different warm-up conditions. HSV, half squat plus whole-body vibrations; HSWV, half squat without whole-body vibrations; REST, rest condition. ${ }^{*} p$ $<0.05$ vs. REST.

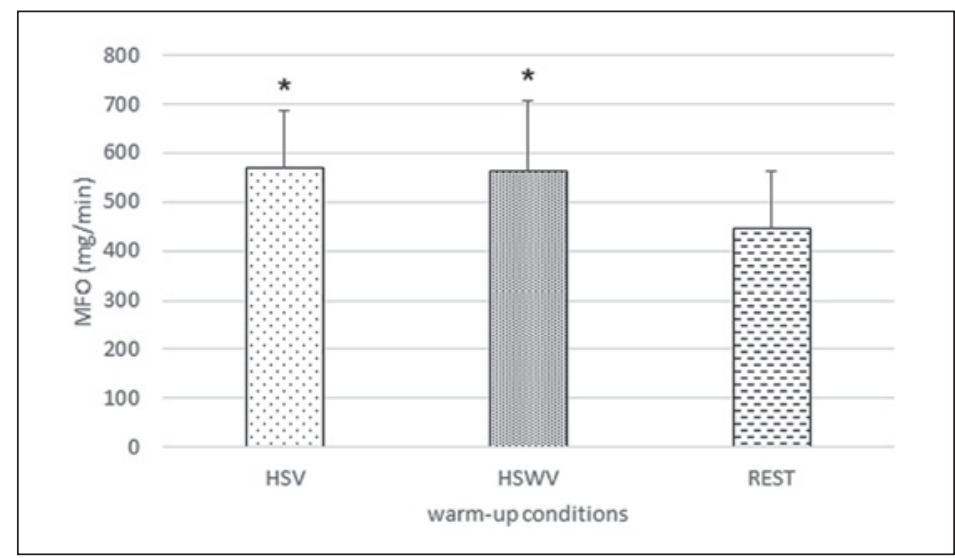

Table 2. Exercise intensities during warm-up conditions and cardiorespiratory variables at peak oxygen uptake and at $\mathrm{FAT}_{\max }$ during the incremental exercise test on a treadmill

\begin{tabular}{lccc}
\hline Variable & HSV & HSWV & REST \\
\hline Warm- $u p$ & & & \\
$\mathrm{HR}_{\text {mean }}, \mathrm{bpm}$ & $106.3 \pm 10.0$ & $100.3 \pm 13.0$ & \\
$\mathrm{HR}$ & & \\
$\mathrm{RPE}$ & $129.4 \pm 12.5$ & $6.6 \pm 11.3$ & \\
\hline Peak & $6.7 \pm 1.8$ & & \\
$\mathrm{VO}_{2}, \mathrm{~mL} / \mathrm{kg} / \mathrm{min}$ & & $25.5 \pm 4.0$ & $24.6 \pm 2.7$ \\
$\mathrm{VO}_{2}, \mathrm{~mL} / \mathrm{min}$ & $24.0 \pm 4.5$ & $2,401.0 \pm 402.9$ & $2,485.6 \pm 486.7$ \\
$\mathrm{HR}_{\text {max }} \%$ & $2,334.2 \pm 528.0$ & $86.0 \pm 5.6$ & $82.5 \pm 7.0$ \\
\hline $\mathrm{FAT}_{\text {max }}$ & $83.8 \pm 7.3$ & & \\
$\mathrm{VO}_{2}, \mathrm{~mL} / \mathrm{kg} / \mathrm{min}$ & & $16.0 \pm 3.1^{*}$ & $13.8 \pm 3.4$ \\
$\mathrm{VO}_{2}, \mathrm{~mL} / \mathrm{min}$ & $15.8 \pm 2.4^{*}$ & $1,561.7 \pm 362.3^{*}$ & $1,343.4 \pm 383.4$ \\
$\mathrm{HR}_{\text {max }} \%$ & $1,532.2 \pm 290.1^{*}$ & $66.3 \pm 7.1^{*}$ & $59.8 \pm 6.3$ \\
\hline
\end{tabular}

Data are presented as mean $\pm \mathrm{SD}$. $\mathrm{HR}_{\text {mean }}$, mean heart rate; $\mathrm{HR}_{\max }$, maximum heart rate; $\mathrm{RPE}$, rate of perceived exertion; $\mathrm{VO}_{2}$, oxygen uptake; $* \leq 0.05$ vs. REST; ${ }^{* *} p \leq 0.01$ vs. REST.

\section{Maximal Fat Oxidation}

MFO was significantly higher in HSV (569.4 $\pm 117.9 \mathrm{mg} / \mathrm{min} ; p=0.013)$ and HSWV (563.8 $\pm 142.9 \mathrm{mg} / \mathrm{min} ; p=0.033$ ) than REST (445.5 $\pm 117.9 \mathrm{mg} / \mathrm{min}$ ) (Fig. 1). At Fat ${ }_{\text {max }}$, oxygen uptake, expressed in $\mathrm{mL} / \mathrm{min}$, was significantly higher in $\mathrm{HSV}(1,532.2 \pm 290.1 \mathrm{~mL} / \mathrm{min} ; p=$ $0.011)$ and $\operatorname{HSWV}(1,561.7 \pm 362.3 \mathrm{~mL} / \mathrm{min} ; p=0.018)$ than REST $(1,343.4 \pm 383.4 \mathrm{~mL} / \mathrm{min})$. No differences were found for $\mathrm{VCO}_{2}$ values among all three conditions.

\section{Lactate Values}

A significant main effect of time $(F=40.851 ; \mathrm{df}=4 ; p<0.01)$ on lactate variables was observed. No significant main effects of the warm-up condition $(F=3.455 ; \mathrm{df}=2 ; p=0.05)$ and warm-up condition $\times$ time interactions on lactate variables were observed. Post hoc analysis showed that lactate levels at T1, T3, T6, and T10 were significantly higher than the PRE point in all conditions. In HSV, lactate values were significantly lower at T10 than T1 and T3. Moreover, lactate values were significantly lower at T6 than T1. In HSWV, lactate values were significantly lower at T10 and T6 than T1. In REST, lactate values were significantly lower at T10 than T1 (Fig. 2). 
Fig. 2. Lactate values at pre warm-up exercise (PRE) and 1 $\min (\mathrm{T} 1), 3 \mathrm{~min}(\mathrm{~T} 3), 6 \mathrm{~min}$ (T6), and 10 min (T10) after the cardiopulmonary exercise test in all three conditions (HSV, half squat plus whole-body vibrations; HSWV, half squat without wholebody vibrations; REST, rest condition). ${ }^{* *} p<0.01$ vs. PRE; ${ }^{a} p<0.01$ vs. T1; ${ }^{\mathrm{b}} p<0.01$ vs. T3; ${ }^{\mathrm{c}} p<0.05$ vs. T1.

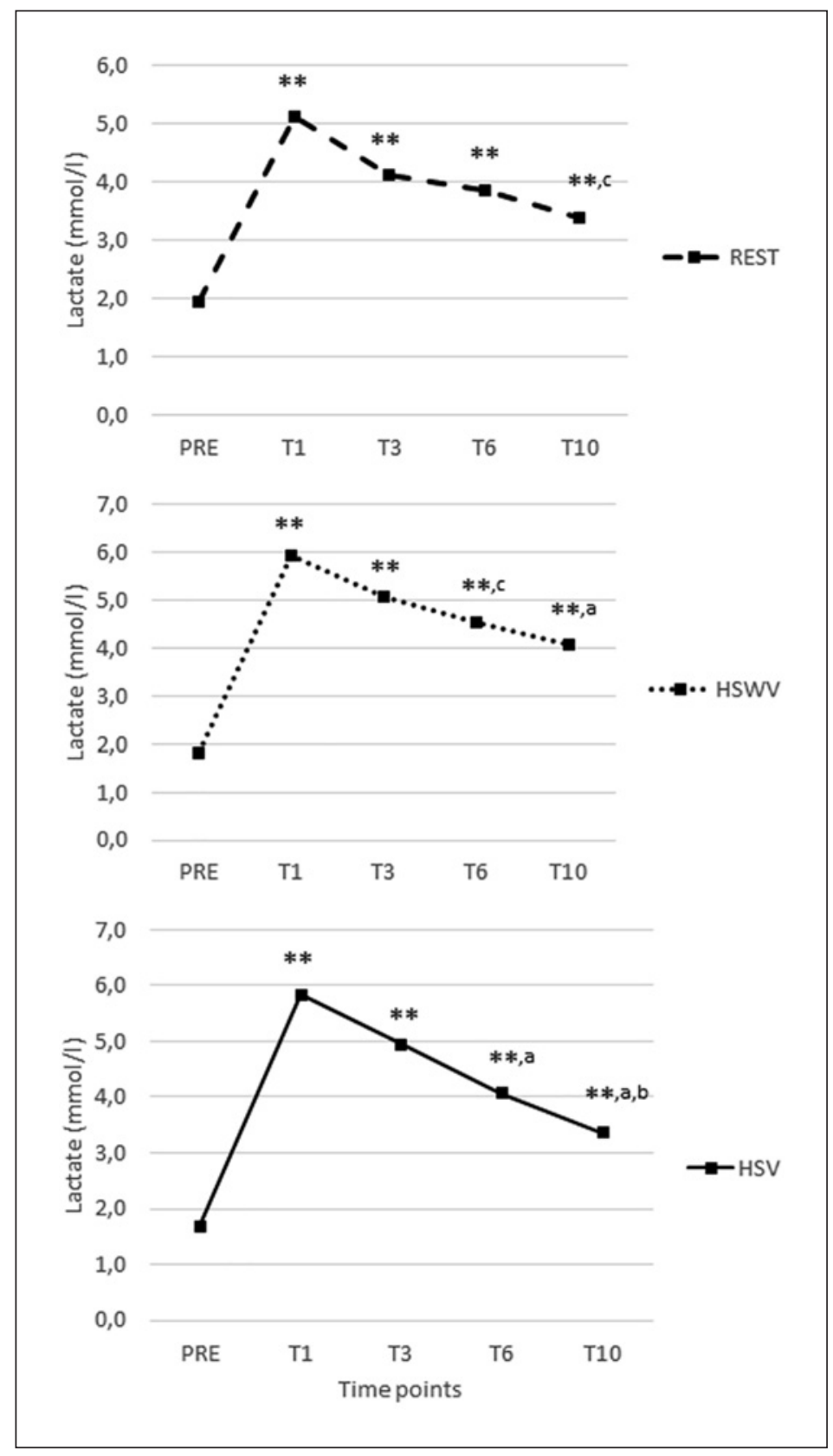

\section{Salivary Cortisol Levels}

Since, to our knowledge, no studies have previously evaluated the effect of WBV on cortisol modifications in relation to MFO in obese subjects, salivary cortisol concentrations were analyzed. The results obtained indicated that no differences in cortisol levels at PRE between all conditions $(\mathrm{REST}=0.785 \pm 0.5 \mathrm{ng} / \mathrm{mL} ; \mathrm{HSV}=0.694 \pm 0.7 \mathrm{ng} / \mathrm{mL} ; \mathrm{HSWV}=0.573$ $\pm 0.225 \mathrm{ng} / \mathrm{mL}$ ) were induced.

Cortisol concentrations were significantly higher in HSV $(1.97 \pm 2.19 \mathrm{ng} / \mathrm{mL} ; p=0.023)$ and HSWV $(1.30 \pm 1.0 \mathrm{ng} / \mathrm{mL} ; p=0.015)$ than REST $(0.62 \pm 0.34 \mathrm{ng} / \mathrm{mL})$ at T1.

In both HSV and HSWV, there was a significant increase of cortisol concentrations after CPET compared to PRE (Fig. 3). In HSV, cortisol peak occurred at T1 (mean peak HSV T1 = 1.9 
Fig. 3. Salivary cortisol concentration in the rest (REST), half squat without vibration (HSWV), and half squat plus vibration (HSV) groups. Histograms represent mean values \pm SEM before (PRE) and after (POST) warm-up, after $1 \mathrm{~min}$ (T1), $10 \mathrm{~min}$ (T10), and $30 \mathrm{~min}$ (Т30) of the cardiopulmonary exercise test. $* p \leq$ 0.05 vs. REST; ${ }^{a} p \leq 0.05$ vs. PRE; ${ }^{\mathrm{b}} p \leq 0.01$ vs. PRE; ${ }^{\mathrm{c}} p \leq 0.01$ vs. POST; ${ }^{\mathrm{d}} p \leq 0.01$ vs. T1; ${ }^{\mathrm{e}} p \leq 0.01$ vs. T10.

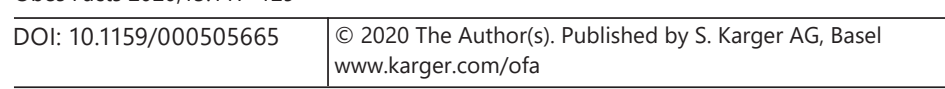

Emerenziani et al.: Whole-Body Vibration and Maximal Fat Oxidation in Obesity
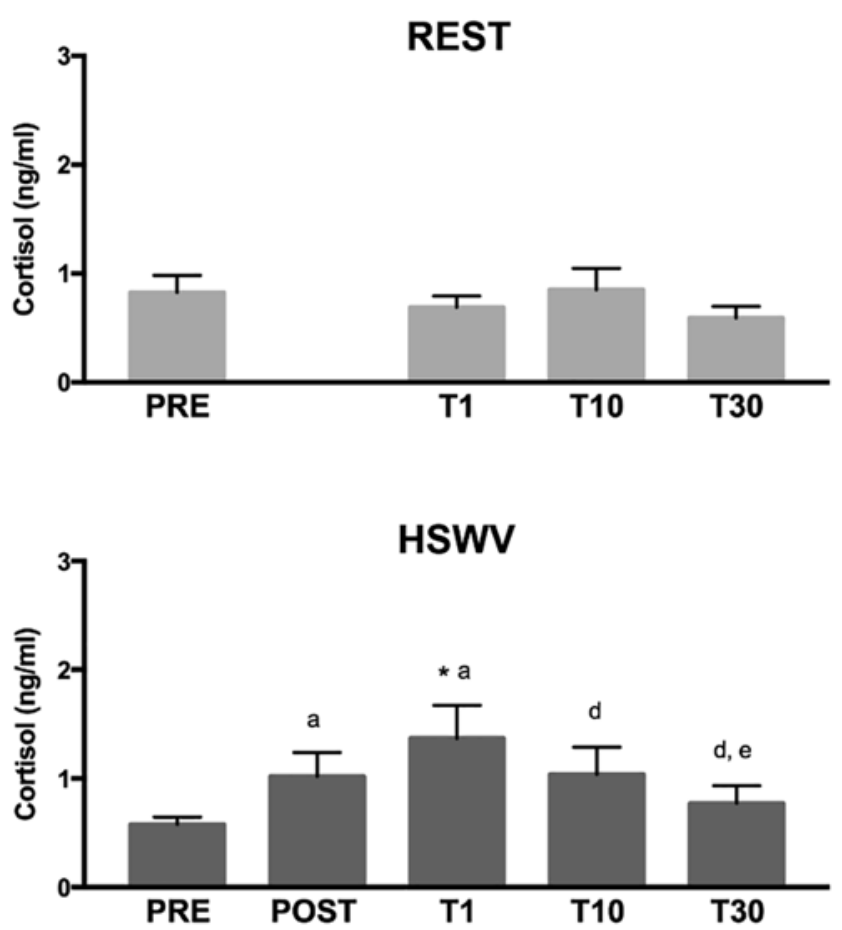

HSV

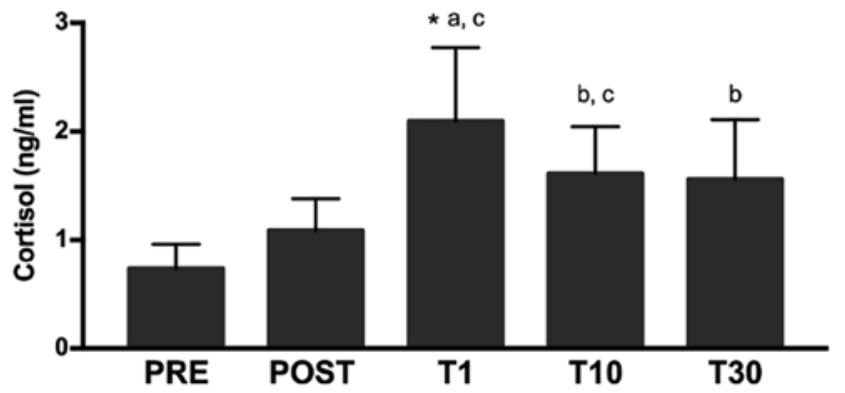

$\pm 2.2 \mathrm{ng} / \mathrm{mL}$ ) and no significant time-dependent reduction was found during recovery phase. Cortisol concentrations were significantly lower at PRE than T1 ( $p=0.028)$, T10 ( $p=0.003)$, and T30 ( $p=0.008)$. Moreover, cortisol concentrations at POST were significantly lower than $\mathrm{T} 1(p=0.003)$ and $\mathrm{T} 10(p=0.006)$.

In HSWV, cortisol levels were significantly higher at POST ( $0.981 \pm 0.7 \mathrm{ng} / \mathrm{mL}, p=0.019)$ and T1 $(1.3 \pm 0.9 \mathrm{ng} / \mathrm{mL} ; p=0.023)$ compared to PRE. Thereafter, a significant, time-dependent reduction was found from T10 to T30 when compared to the T1 point (T10 $=0.992 \pm 0.8 \mathrm{ng}$ / $\mathrm{mL} ; p=0.004$ and T30 $=0.743 \pm 0.5 \mathrm{ng} / \mathrm{mL} ; p=0.003$ ). Moreover, cortisol levels were significantly lower at T30 than T10 ( $p=0.002)$, and no statistically significant differences were found among the other time points. 
Emerenziani et al.: Whole-Body Vibration and Maximal Fat Oxidation in Obesity

\section{Discussion/Conclusion}

This study evaluated the effects of WBV on MFO in obese adult men. Our study shows that the addition of WBV stimulus to an active warm-up did not enhance MFO during CPET in obese male adults. However, performing an active warm-up, with or without the vibration stimulus, resulted in an increased MFO compared to not performing any warm-up. Moreover, cortisol concentration tends to remain higher during recovery phase when subjects performed a warm-up with vibration stimulus. To our knowledge, this is the first study which evaluated the effect of WBV on MFO in obese subjects and modification of cortisol level upon different warm-up conditions.

The new European guidelines for adult obesity management [26] suggest that $5-10 \%$ of weight loss is enough to obtain substantial health benefits such as decrease in comorbidities [26]. Furthermore, it has been demonstrated that subjects that increase their level of PA over time have a decreased mortality rate compared to those who remain unfit [27].

Indeed, regular PA provides health benefits, and it is considered an essential component of primary and secondary preventive actions for most of metabolic syndrome-related pathologies [26]. Therefore, exercise training should be organized and personalized in obese subjects in order to maximize fat oxidation and increase subjects' physical fitness and modify subjects' body composition.

WBV training has been recognized as an alternative exercise training to improve physical fitness also in obese subjects $[10,12,13]$. WBV stimulus is applied through a vibration platform which delivers high-frequency mechanical stimuli $(\mathrm{Hz})$ with a small amplitude (mm). Interestingly, WBV induces both muscle activation and hormonal responses during either static or dynamic exercises in obese adults [14, 16, 28, 29]. Moreover, WBV has been confirmed as potential alternative exercise training method to increase physical fitness adherence in non-obese [11] and obese adults [10]. As previously reported, WBV might have positive acute and chronic effects in both obese mice [30] and humans [28, 10]. Huang et al. [30] showed that WBV could improve exercise performance and prevent fat accumulation in obese mice suggesting a possible clinical use of WBV in the prevention and treatment of obesity. Giunta et al. [16] studied the acute effects of WBV alone, or in combination with squatting, on GH levels and lactate concentrations in obese women, showing that WBV alone might stimulate GH release and lactate production. GH is an anabolic hormone, and its metabolic effects imply the stimulation of the release and oxidation of free fatty acids with the aim to decrease glucose and protein oxidation [31]. Therefore, we might speculate that WBV practice might have anabolic effects stimulating GH release and increase MFO in obese adults. Our results showed that there was no significant effect of WBV on MFO, and therefore we cannot confirm the hypothetical relationship between GH release and MFO in obese adult male. This lack of relationship was also confirmed in young men with normal body weight [32]. In fact, Hansen et al. [32] showed that GH administration combined with aerobic exercise increased lipolytic parameters substantially more than exercise alone but did not further augment whole-body fat oxidation. This result is in line with the crossover concept developed by Brooks and Mercier [33], which affirms that during exercise glycolysis in human skeletal muscles dominates and downregulates fatty acid entry into the citric acid cycle.

In our study, MFO was higher when subjects performed both the active warm-up (with or without WBV) than the control condition (REST). However, no differences in MFO during CPET after the two active warm-up conditions were observed. Therefore, WBV did not further augment whole-body fat oxidation. We might affirm that active warm-up, rather than WBV, increasing the GH release, results in higher fat oxidation during the following exercise. The active warm-up involves exercise and is likely to induce greater metabolic and cardiovascular changes. In detail, warm-up practice might increase oxygen delivery to the muscle, and conse- 
Emerenziani et al.: Whole-Body Vibration and Maximal Fat Oxidation in Obesity

quently increase aerobic contribution and decrease oxygen deficit [34]. Therefore, warm-up may allow subsequent tasks to start with an elevated baseline of $\mathrm{VO}_{2}$ and consequently stimulates the aerobic metabolism. These positive effects are also confirmed in our study. In fact, $\mathrm{VO}_{2}$ and $\% \mathrm{HR}_{\max }$ at $\mathrm{Fat}_{\text {max }}$, and MFO were significantly higher when CPET was performed after active warm-up (HSV or HSWV) than no warm-up (REST). It is interesting to notice that mean HR during warm-up were not different between HSV and HSWV, indicating that WBV did not increase the mean effort during squatting exercise. Differently, $\mathrm{HR}_{\max }$ was significantly higher in HSV than HSWV, indicating a higher peak of HR during HSV warm-up.

Regarding the graded incremental exercise test, even if cycle ergometer has been more often used for CPET tests in obese subjects, we chose the treadmill because it reflects better the walking exercise that subjects might perform as regular unsupervised PA in an almost daily routine. Our MFO results were higher than those reported by other studies in obese subjects (MFO ranging from 0.25 to $0.4 \mathrm{~g} / \mathrm{min}$ ) $[9,35]$. These differences might by justified by the different graded exercise protocol, and nutritional status of the subjects during the experimental test. In fact, scientific evidence [8] showed that fat oxidation rates were $28 \%$ higher when walking compared with cycling in moderately trained male subjects. In our study, subjects were obese, and they performed an incremental exercise test on the treadmill at least $1.5 \mathrm{~h}$ after breakfast. Therefore, these conditions might influence the MFO in our subjects resulting in a higher MFO compared with other studies.

The non-significant differences in lactate values between the three warm-up conditions might indicate that the anaerobic sources during CPET were the same independent of the characteristics of the warm-up. In the REST condition, lactate values were slightly lower than HSV and HSWV conditions. Once again, these results might be justified by the positive effects of active warm-up on energy substrate utilization.

Regarding the cortisol variable, our results showed that active warm-up independent of WBV stimulus might not influence cortisol responses to CPET. However, when CPET is preceded by warm-up plus WBV, cortisol concentration tends to remain high during the recovery phase. In contrast, when CPET was preceded by the HSWV and REST warm-up conditions, cortisol concentration tended to return to the baseline values after $30 \mathrm{~min}$ from the end of the test. It is worth noting that the HPA axis is influenced by psychological and physical stress, depending on the type, intensity, and duration of the stressor and individual characteristics [36]. Scientific evidence showed that combining WBV with other types of exercise, including half squat or external load might maximize GH and cortisol release in healthy [37] and obese subjects [38]. Other studies reported conflicting results on the effects of acute WBV on HPA in healthy and non-healthy subjects [28, 37, 38]. These differences might be justified by the different WBV protocol and by the individual characteristics. For instance, HPA response might be altered in obese subjects. We are unable to draw definitive conclusions about cortisol results, but we could speculate that WBV practice influences the cortisol concentration during the recovery phase after exercise.

In conclusion, no additional increase in MFO during CPET was observed when active warm-up was performed with the WBV stimulus. However, MFO rates were significantly higher when CPET was preceded by active warm-up than no warm-up. When CPET was performed after an active warm-up with WBV stimulus, cortisol concentration tended to remain high during the recovery phase, indicating a long effect of WBV on cortisol concentrations.

We are aware that different metabolic and hormonal background might influence individual MFO and hormonal responses to the three different warm-up conditions. In our cohort, even if individual mean metabolic and hormonal parameters were within the normal range, 5 subjects had insulin resistance and just one of them had diabetes diagnosis. Moreover, as expected, 3 subjects had low testosterone levels, and 2 subjects had low IGF-1 levels. However, 
MFO was lower in the REST than the WBV and WWBV conditions in subjects with metabolic and hormonal alterations, showing that this different physical background did not influence the responses to the three different warm-up conditions. Future studies will focus on the evaluation of the effects of WBV on MFO in subjects with different metabolic and hormonal conditions.

Further studies are mandatory to determine the effects of active warm-up plus WBV on MFO during a following continuous aerobic exercise lasting more than $30 \mathrm{~min}$ and to investigate in depth the potential effects of WBV on the management of obese subjects.

\section{Acknowledgement}

We thank Lavinia Falcioni and Lorenzo Innocenti for their help in evaluating the PA of the subjects analyzed in the study. Moreover, we are grateful to all subjects who participated in the study.

\section{Statement of Ethics}

This study was conducted according to the Declaration of Helsinki and approved by the Sapienza University of Rome Ethical Committee (approval No. 70/11, 2011).

\section{Disclosure Statement}

The authors have no conflicts of interest to declare.

\section{Funding Sources}

This research was partially funded by: MIUR grants PRIN, 2015-2015JSWLTN_004 to C. Baldari; FFABR and Italian Ministry of Research and PRIN 2017 grant No. 2017FJSM9S to G.P. Emerenziani; PON-MIUR 03PE000_78_2; Ministry of Health GR-2013-02357959 to E.A. Greco. The funders had no role in study design, data collection and analysis, decision to publish, or preparation of the manuscript.

\section{Author Contributions}

G.P. Emerenziani, S. Migliaccio, and L. Guidetti designed the study. D. Ferrari, E.A. Greco, F. Perroni, and C. Marocco assisted with data collection. G.P. Emerenziani, S. Fittipaldi, and V.M. Bimonte analyzed and interpreted the data. G.P. Emerenziani and S. Fittipaldi were major contributors in writing the manuscript. A. Lenzi and C. Baldari contributed to the data evaluation and manuscript preparation. All authors read and approved the final version of the manuscript. 


\section{References}

1 World Obesity Federation. Key facts. WHO. 16 February 2018. Available from: http://www.who.int/newsroom/fact-sheets/detail/obesity-and-overweight

2 Adams KF, Schatzkin A, Harris TB, Kipnis V, Mouw T, Ballard-Barbash R, et al. Overweight, obesity, and mortality in a large prospective cohort of persons 50 to 71 years old. N Engl J Med. 2006 Aug;355(8):763-78.

3 Annesi JJ, Vaughn LL. Directionality in the Relationship of Self-regulation, Self-efficacy, and Mood Changes in Facilitating Improved Physical Activity and Nutrition Behaviors: Extending Behavioral Theory to Improve Weight-Loss Treatment Effects. J Nutr Educ Behav. 2017 Jun;49(6):505-512.e1.

4 Emerenziani GP, Gallotta MC, Migliaccio S, Ferrari D, Greco EA, Saavedra FJ, et al. Effects of an individualized home-based unsupervised aerobic training on body composition and physiological parameters in obese adults are independent of gender. J Endocrinol Invest. 2018 Apr;41(4):465-73.

5 Jeukendrup AE, Wallis GA. Measurement of substrate oxidation during exercise by means of gas exchange measurements. Int J Sports Med. 2005 Feb;26 Suppl 1:S28-37.

6 Pérez-Martin A, Dumortier M, Raynaud E, Brun JF, Fédou C, Bringer J, et al. Balance of substrate oxidation during submaximal exercise in lean and obese people. Diabetes Metab. 2001 Sep;27(4 Pt 1):466-74.

7 van Aggel-Leijssen DP, Saris WH, Wagenmakers AJ, Senden JM, van Baak MA. Effect of exercise training at different intensities on fat metabolism of obese men. J Appl Physiol (1985). 2002 Mar; 92(3):1300-9.

8 Achten J, Venables MC, Jeukendrup AE. Fat oxidation rates are higher during running compared with cycling over a wide range of intensities. Metabolism. 2003 Jun;52(6):747-52.

9 Maunder E, Plews DJ, Kilding AE. Contextualising Maximal Fat Oxidation During Exercise: Determinants and Normative Values. Front Physiol. 2018 May;23:9:599.

10 Zago M, Capodaglio P, Ferrario C, Tarabini M, Galli M. Whole-body vibration training in obese subjects: A systematic review. PLoS One. 2018 Sep;13(9):e0202866.

11 Emerenziani GP, Meucci M, Gallotta MC, Buzzachera CF, Guidetti L, Baldari C. Whole body vibration: unsupervised training or combined with a supervised multi-purpose exercise for fitness? J Sports Sci. 2014;32(11): 1033-41.

12 Nam SS, Sunoo S, Park HY, Moon HW. The effects of long-term whole-body vibration and aerobic exercise on body composition and bone mineral density in obese middle-aged women. J Exerc Nutrition Biochem. 2016 Jun;20(2):19-27.

13 Severino G, Sanchez-Gonzalez M, Walters-Edwards M, Nordvall M, Chernykh O, Adames J, et al. Whole-Body Vibration Training Improves Heart Rate Variability and Body Fat Percentage in Obese Hispanic Postmenopausal Women. J Aging Phys Act. 2017 Jul;25(3):395-401.

14 Cardinale M, Soiza RL, Leiper JB, Gibson A, Primrose WR. Hormonal responses to a single session of wholebody vibration exercise in older individuals. Br J Sports Med. 2010 Mar;44(4):284-8.

15 Rigamonti AE, Haenelt M, Bidlingmaier M, De Col A, Tamini S, Tringali G, et al. Obese adolescents exhibit a constant ratio of GH isoforms after whole body vibration and maximal voluntary contractions. BMC Endocr Disord. 2018 Dec;18(1):96.

16 Giunta M, Cardinale M, Agosti F, Patrizi A, Compri E, Rigamonti AE, et al. Growth hormone-releasing effects of whole body vibration alone or combined with squatting plus external load in severely obese female subjects. Obes Facts. 2012;5(4):567-74.

17 Lange KH. Fat metabolism in exercise-with special reference to training and growth hormone administration. Scand J Med Sci Sports. 2004 Apr;14(2):74-99.

18 Bak JF, Møller N, Schmitz O. Effects of growth hormone on fuel utilization and muscle glycogen synthase activity in normal humans. Am J Physiol. 1991 May;260(5 Pt 1):E736-42.

19 Bernstein RS, Thornton JC, Yang MU, Wang J, Redmond AM, Pierson RN Jr, et al. Prediction of the resting metabolic rate in obese patients. Am J Clin Nutr. 1983 Apr;37(4):595-602.

20 Ainsworth BE, Bassett DR Jr, Strath SJ, Swartz AM, O’Brien WL, Thompson RW, et al. Comparison of three methods for measuring the time spent in physical activity. Med Sci Sports Exerc. 2000 Sep;32(9 Suppl):S45764.

21 Nieman DC, Austin MD, Dew D, Utter AC. Validity of COSMED’s quark CPET mixing chamber system in evaluating energy metabolism during aerobic exercise in healthy male adults. Res Sports Med. 2013;21(2):136-45.

22 Balasekaran G, Loh MK, Govindaswamy VV, Cai SJ. Omni Scale Perceived Exertion responses in obese and normal weight male adolescents during cycle exercise. J Sports Med Phys Fitness. 2014 Apr;54(2):186-96.

23 Venables MC, Jeukendrup AE. Endurance training and obesity: effect on substrate metabolism and insulin sensitivity. Med Sci Sports Exerc. 2008 Mar;40(3):495-502.

24 Franckowiak SC, Dobrosielski DA, Reilley SM, Walston JD, Andersen RE. Maximal heart rate prediction in adults that are overweight or obese. J Strength Cond Res. 2011 May;25(5):1407-12.

25 Achten J, Gleeson M, Jeukendrup AE. Determination of the exercise intensity that elicits maximal fat oxidation. Med Sci Sports Exerc. 2002 Jan;34(1):92-7.

26 Durrer Schutz D, Busetto L, Dicker D, Farpour-Lambert N, Pryke R, Toplak H, et al. European Practical and Patient-Centred Guidelines for Adult Obesity Management in Primary Care. Obes Facts. 2019;12(1):40-66.

27 Wannamethee SG, Shaper AG, Walker M. Changes in physical activity, mortality, and incidence of coronary heart disease in older men. Lancet 1998 May;351(9116):1603-8. 
28 Elmantaser M, McMillan M, Smith K, Khanna S, Chantler D, Panarelli M, et al. A comparison of the effect of two types of vibration exercise on the endocrine and musculoskeletal system. J Musculoskelet Neuronal Interact. 2012 Sep;12(3):144-54.

29 Alvarez-Alvarado S, Jaime SJ, Ormsbee MJ, Campbell JC, Post J, Pacilio J, et al. Benefits of whole-body vibration training on arterial function and muscle strength in young overweight/obese women. Hypertens Res. 2017 May;40(5):487-92.

30 Huang CC, Tseng TL, Huang WC, Chung YH, Chuang HL, Wu JH. Whole-body vibration training effect on physical performance and obesity in mice. Int J Med Sci. 2014 Sep;11(12):1218-27.

31 Møller N, Jørgensen JO. Effects of growth hormone on glucose, lipid, and protein metabolism in human subjects. Endocr Rev. 2009 Apr;30(2):152-77.

32 Hansen M, Morthorst R, Larsson B, Dall R, Flyvbjerg A, Rasmussen MH, et al. No effect of growth hormone administration on substrate oxidation during exercise in young, lean men. J Physiol. 2005 Sep;567(Pt 3): 1035-45.

33 Brooks GA, Mercier J. Balance of carbohydrate and lipid utilization during exercise: the "crossover" concept. J Appl Physiol (1985). 1994 Jun;76(6):2253-61.

34 Guidetti L, Emerenziani GP, Gallotta MC, Baldari C. Effect of warm up on energy cost and energy sources of a ballet dance exercise. Eur J Appl Physiol. 2007 Feb; 99(3):275-81.

35 Dandanell S, Præst CB, Søndergård SD, Skovborg C, Dela F, Larsen S, et al. Determination of the exercise intensity that elicits maximal fat oxidation in individuals with obesity. Appl Physiol Nutr Metab. 2017 Apr; 42(4):405-12.

36 Singh A, Petrides JS, Gold PW, Chrousos GP, Deuster PA. Differential hypothalamic-pituitary-adrenal axis reactivity to psychological and physical stress. J Clin Endocrinol Metab. 1999 Jun;84(6):1944-8.

37 Giunta M, Rigamonti AE, Agosti F, Patrizi A, Compri E, Cardinale M, et al. Combination of external load and whole body vibration potentiates the GH-releasing effect of squatting in healthy females. Horm Metab Res. 2013 Aug;45(8):611-6.

38 Rigamonti AE, De Col A, Tamini S, Tringali G, De Micheli R, Abbruzzese L, et al. GH responses to whole body vibration alone or in combination with maximal voluntary contractions in obese male adolescents. Growth Horm IGF Res. 2018 Oct-Dec;42-43:22-7. 